\title{
Reliability analysis of aerostatic instability of suspension bridge under stochastic wind loading on Bayesian theory
}

\author{
Wang Huasheng ${ }^{1,}$ a * \\ ${ }^{1}$ Inner Mongolia Communications Planningand Design Institute Co., Ltd, \\ Huhhot, Inner Mongolia, China \\ atongjidfh@163.com
}

Keywords: Bayesian theory, Extreme value wind speed, Limit state function, Aerostatic instability, Reliability index.

\begin{abstract}
Based on the structural reliability theory, the extreme wind speed forecast method was used to establish the reliability model for analyzing aerostatic instability of long-span suspension bridge. The limit state equation of reliability analysis model is a function of conversion factor, critical wind speed of the aerostatic instability, gust factor and extreme wind speed at the bridge site. In this paper, JC method based on the first order second moment reliability theory was used to calculate reliability indices of the aerostatic instability of the suspension bridge. The results indicate that probability assessment and reliability analysis of aerostatic instability of suspension bridge under stochastic wind loading based on Bayesian theory are more precise and practical, which is more valuable in engineering application.
\end{abstract}

\section{Introduction}

With the ever-growing span-length of bridges, aerostatic instability is becoming a matter of significant design consideration for long-span suspension bridges again. Aerostatic instability takes place when bridges are exposed to wind speeds beyond a certain critical value that could be got by wind tunnel experiments and theoretical calculations with tests-obtained parameters. Nevertheless, because most of these parameters from the prediction are actually indefinite variables or/and empirically assumed values from researchers due to lack of complete theory, it is more rational to conduct an analysis of probabilistic reliability to determine the probability of the bridge failure resulting from aerostatic instability for a given return period rather than stating a single critical wind speed [1].

In this paper, a reliability analysis model was established by taking account of four random and mutually independent variables, and safety margin, also a random variable, which only depends on stochastic nature of these variables [1]. The forecast method of extreme wind speed is introduced in the present paper, which aims to obtain the true value of wind speed at the bridge site because the samples of wind speed are always not enough. Based on the wind speed of Bayesian estimates, the reliability indices of aerostatic instability for four long-span cable-stayed bridges were calculated and compared to the results of maximum likelihood estimates.

\section{The Gumbel Model}

The extreme value of wind speed is for Gumbel (extreme value of type-I) distribution [2, 3] in reliability analysis of aerostatic instability. For the Gumbel model, the probability distribution function (PDF) and the cumulative distribution function (CDF) of the wind speed $\mathrm{T}$ are given respectively, by

$$
f(t)=\frac{1}{\sigma} e^{\left(-\frac{t-\mu}{\sigma}-e^{\frac{t-\mu}{\sigma}}\right)}, F(t)=\exp \left\{-\exp \left[-\left(\frac{t-\mu}{\sigma}\right)\right]\right\},-\infty<t, \mu<\infty, \sigma>0
$$

where, $\sigma$ and $\mu$ are the scale parameters and location [4], respectively. 
The wind speed forecast value which recurrence is $T$ years (assurance rate is $1-\frac{1}{T}$ ) can be obtained by the logarithmic form of function (2)

$$
t_{\frac{1}{T}}=\mu-\sigma\left\{\ln \left[-\ln \left(1-\frac{1}{T}\right)\right]\right\} .
$$

\section{Bayesian Theory}

In the Bayesian method, we regard $\mu$ and $\sigma$ behaving as random variables with a joint PDF $\pi(\mu, \sigma)$ [4]. For extreme value of Gumbel distribution, the Jeffrey's non-informative prior [5, 6] is given by

$$
\pi(\mu, \sigma)=\frac{1}{\sigma^{2}} .
$$

To evaluate the expression above to acquire approximate Bayesian estimates of $t_{\frac{1}{T}}$, we would use Lindley's approximation method [6-8].

\section{Lindley Approximation}

Let

$$
I=\frac{\int u(\theta) v(\theta) e^{L(\theta)} d \theta}{\int v(\theta) e^{L(\theta)} d \theta} .
$$

where, $\theta=\left(\theta_{1}, \theta_{2}, \ldots, \theta_{k}\right)$, a vector of parameters. Also, let $\mathrm{L}=\mathrm{Log}$ (likelihood function). Note that Iis the posterior expectation of $u(\theta)$ given the failure data $v(\theta)$. Denote by

$$
\begin{aligned}
& u_{1}=\frac{\partial u}{\partial \theta_{1}}, u_{2}=\frac{\partial u}{\partial \theta_{2}}, u_{11}=\frac{\partial^{2} u}{\partial \theta_{1}^{2}}, u_{22}=\frac{\partial^{2} u}{\partial \theta_{2}^{2}} . \\
& p=\pi\left(\theta_{1}, \theta_{2}\right), p_{1}=\frac{\partial p}{\partial \theta_{1}}, p_{2}=\frac{\partial p}{\partial \theta_{2}} . \\
& L_{20}=\frac{\partial^{2} L}{\partial \theta_{1}^{2}}, L_{02}=\frac{\partial^{2} L}{\partial \theta_{2}^{2}}, L_{30}=\frac{\partial^{3} L}{\partial \theta_{1}^{3}}, L_{03}=\frac{\partial^{3} L}{\partial \theta_{2}^{3}} . \\
& \sigma_{11}=\left(-L_{20}\right)^{-1}, \sigma_{22}=\left(-L_{02}\right)^{-1} .
\end{aligned}
$$

and

$$
\begin{aligned}
& E(u(\theta) \mid \vec{t})=u\left(\overline{\theta_{1}}, \overline{\theta_{2}}\right)+\frac{1}{2}\left(u_{11} \sigma_{11}+u_{22} \sigma_{22}\right)+p_{1} u_{1} \sigma_{11}+p_{2} u_{2} \sigma_{22} \\
& +\frac{1}{2}\left(L_{30} u_{1} \sigma_{11}^{2}+L_{03} u_{2} \sigma_{22}^{2}+L_{21} u_{2} \sigma_{11} \sigma_{22}+L_{12} u_{1} \sigma_{22} \sigma_{11}\right)
\end{aligned}
$$

where, $\overline{\theta_{1}}$ and $\overline{\theta_{2}}$ are the classical MLEs for $\theta_{1}$ and $\theta_{2}$, respectively [6].

\section{Forecast Model of Extreme Value Wind Speed}

Thus, a Bayesian approximate estimate for $\overline{t_{B}}$ is given by

$$
\overline{t_{B}}=\bar{\mu}+4.6 \bar{\sigma}+p_{2} u_{2} \sigma_{22}+\frac{1}{2}\left(L_{30} \sigma_{11}^{2}+L_{03} u_{2} \sigma_{22}^{2}+L_{21} u_{2} \sigma_{11} \sigma_{22}+L_{12} u_{1} \sigma_{22} \sigma_{11}\right) \text {. }
$$


where, $\bar{\mu}$ and $\bar{\sigma}$ are the classical MLEs for $\mu$ and $\sigma$, respectively.

\section{Numerical Analysis}

In this section, a numerical study is presented to compare the maximum likelihood and Bayes estimates for determining the wind speed model subject to specified reliability. The numerical simulation was carried out in the following manner [6]:

Because of the size of the simulation, some of all the numerical results are listed in table 1 under 99\% reliability. In Table 1 , the size of the prior sample used to calculate the Bayes estimate $\mu_{B}$ was presented. $t_{\frac{1}{T}}$ is the true wind speed, $\bar{t}_{\frac{1}{T}}$ is the ML estimates of wind speed, $\bar{t}_{B}$ is the Bayes estimation value of wind speed, $\left|t_{\frac{1}{T}}-\bar{t}_{\frac{1}{T}}\right| / t_{\frac{1}{T}}$ and $\left|t_{\frac{1}{T}}-\bar{t}_{B}\right| / t_{\frac{1}{T}}$ represent the absolute value of the difference between the true wind speed, and maximum likelihood and Bayes wind speed estimates, respectively.

Table 1. Numerical Study of the Gumbel wind speed.

\begin{tabular}{cccccc|c|cc}
\hline$\mu_{B}$ & $\bar{\mu}$ & $\sigma_{B}$ & $\bar{\sigma}$ & $t_{\alpha}$ & $\bar{t}_{\alpha}$ & $\bar{t}_{B}$ & $\left|t_{\frac{1}{T}}-\bar{t}_{\frac{1}{T}}\right| / t_{\frac{1}{T}}$ & $\left|t_{\frac{1}{T}}-\bar{t}_{B}\right| / t_{\frac{1}{T}}$ \\
\hline 14.9081 & 15.1877 & 2 & 1.9201 & 24.2 & 24.0202 & 24.3331 & $0.74 \%$ & $0.55 \%$ \\
\hline
\end{tabular}

In Table 1, the Bayes estimate is closer to the true wind speed than the maximum likelihood.

\section{Engineering Application}

Based on aerostatic instability model from [9], wind speed is obtained by the Bayesian method, and the reliability indices of Xihoumen Bridgeis was calculated by the JC method.

Limit State Function. A limit state function can be represented by

$$
G=C_{w} U_{f}-G_{s} U_{b} .
$$

in which $C_{w}$ is the wind conversion factor, and $U_{f}$ is the basic aerostatic instability speed, and $G_{s}$ is the gust speed factor, and $U_{b}$ is the basic wind speed at the bridge deck location.

The statistics of $C_{w}, U_{f}, G_{s}$ and $U_{b}$ of Xihoumen Bridge are shown in Table 2 [1,10].

Table 2. Random variables and their statistical properties of Xihoumen Bridge - $\mathrm{L}=1650 \mathrm{~m}$.

\begin{tabular}{|c|c|c|c|}
\hline Random variables & Mean & Coefficientsof variation & Distribution type \\
\hline$C_{w}$ & 1 & 0.1 & Normal \\
\hline$U_{f}$ & $\begin{array}{c}194 \\
\text { (empirical formula) } \\
115 \\
\text { (finite element) } \\
95 \\
\text { (wind tunnel test) }\end{array}$ & 0.1 & Lognormal \\
\hline$G_{s}$ & 1.2 & 0.12 & Normal \\
\hline$U_{b}$ & $\begin{array}{c}23.17 \\
\text { (Bayes estimates) } \\
24.23 \\
\text { (ML estimates) }\end{array}$ & 0.3 & Extreme type I \\
\hline
\end{tabular}


Reliability Analysis of Four Cable-Stayed Bridges. On the basis of the JC approach, the reliability index $\beta$ are numerically listed in Table 3 and compared to the traditional safety factors defined as $K=\left[U_{c r}\right] / \mu_{G} U_{d}[11]$, which listed in Table 4.

Table 3. Reliability indices of suspension bridge.

\begin{tabular}{ccccccc}
\hline \multirow{2}{*}{ Bridge name } & \multicolumn{2}{c}{ empirical formula } & \multicolumn{2}{c}{ Finite element } & \multicolumn{2}{c}{ Wind tunnel test } \\
\cline { 2 - 7 } & Bayes & ML & Bayes & ML & Bayes & ML \\
\hline Xihoumen Bridge & 7.38 & 7.51 & 6.22 & 6.43 & 5.11 & 5.36 \\
\hline
\end{tabular}

Table 4. Safety factors of suspension bridge.

\begin{tabular}{ccccccc}
\hline \multirow{2}{*}{ Bridge name } & \multicolumn{2}{c}{ empirical formula } & \multicolumn{2}{c}{ Finite element } & \multicolumn{2}{c}{ Wind tunnel test } \\
\cline { 2 - 6 } & Bayes & ML & Bayes & ML & Bayes & ML \\
\hline Xihoumen Bridge & 2.93 & 3.06 & 1.74 & 1.82 & 1.44 & 1.51 \\
\hline
\end{tabular}

It can be concluded from Table 3 and 4 that the reliability indices of Bayes estimates are smaller than those of ML estimates for Xihoumen Bridge, which are all same as the safety factors of aerostatic stability, which indicates that the results of ML estimation are unsafe while the Bayes estimation could give the rational calculation results.

\section{Conclusion}

In this paper, a forecast method based on Bayesian theory of wind speed was introduced, upon which the reliability analysis model was established. The reliability indices were calculated using the JC approach based on the empirical formula, finite element analysis and wind tunnel test, in which the results of Bayes estimates and ML estimates were compared and indicate that probability assessment and reliability analysis of aerostatic instability of suspension bridge under stochastic wind loading based on Bayesian theory are more precise and scientific, which is significantly valuable in the application of practical engineering projects.

\section{References}

[1] Y. J. Ge, H. F. Xiang, H. Tanaka, Application of a reliability analysis model to bridge flutter under extreme winds. J. Wind Eng. Ind. Aerodyn, 28 (2000) 155-167.

[2] M. Kang, K. Ko, J. Huh, Determination of extreme wind values using the gumbel distribution. Energy, 86 (2015) 51-58.

[3] I. Vidal, A Bayesian analysis of the Gumbel distribution: an application to extreme rainfall data. Stoch. Environ. Res. Risk. Assess. 28(3) (2014) 571-582.

[4] B. Miladinovic, Kernel density estimation of reliability with applications to extreme value distribution. University of South Florida, (2008).

[5] M. Han, Bayesian Statistical and Its Applications. Tongji University Press, SHH. (2015).

[6] B. Miladinovic, C. P. Tsokos, Ordinary, bayes, empirical bayes, and non-parametric reliability analysis for the modified gumbel failure model. Nonlin. Anal. 71 (2009) 1426-1436.

[7] C. B. Guure, N. A. Ibrahim, Approximate bayesian estimates of weibull parameters with Lindley's method. Sains. Malaysiana, 43(9) (2014) 1433-1437.

[8] S. Ali, On the bayesian estimation of the weighted Lindley distribution. J. Statist. Comput. Simul. 85(5) (2015) 855-880.

[9] Y. J. Ge, Wind-induced Reliability Theory and Its Applications of Bridge Structures. Tongji 
University, SHH, (1997).

[10] Y. J. Ge, Wind resistance of long span suspension bridge, China Communications press, Beijing, China, (2011).

[11] MOT: Ministry of Transport of the People's Republic of China. Wind-resistent Design Specification for Highway Bridges, JTG/T D60-01, (2004). 